\title{
Neonatal and infant outcome in boys and girls born very prematurely
}

\author{
Janet L. Peacock', Louise Marston ${ }^{2}$, Neil Marlow ${ }^{3}$, Sandra A. Calvert ${ }^{4}$ and Anne Greenough ${ }^{5}$
}

INTRODUCTION: Although important new strategies have improved outcomes for very preterm infants, males have greater mortality/morbidity than females. We investigated whether the excess of adverse later effects in males operated through poorer neonatal profile or if there was an intrinsic male effect.

RESULTS: Male sex was significantly associated with higher birth weight, death or oxygen dependency ( $72 \%$ vs. $61 \%$, boys vs. girls), hospital stay (97 vs. 86 days), pulmonary hemorrhage (15\% vs. $10 \%)$, postnatal steroids (37\% vs. $21 \%)$, and major cranial ultrasound abnormality ( $20 \%$ vs. $12 \%$ ). Differences remained significant after adjusting for birth weight and gestation. At follow-up, disability, cognitive delay, and use of inhalers remained significant after further adjustment.

DISCUSSION: We conclude that in very preterm infants, male sex is an important risk factor for poor neonatal outcome and poor neurological and respiratory outcome at follow-up. The increased risks at follow-up are not explained by neonatal factors and lend support to the concept of male vulnerability following preterm birth.

METHODS: Data came from the United Kingdom Oscillation Study, with 797 infants (428 boys) born at 23-28 wk gestational age. Thirteen maternal factors, 8 infant factors, 11 acute outcomes, and neurological and respiratory outcomes at followup were analyzed. Follow-up outcomes were adjusted for birth and neonatal factors sequentially to explore mechanisms for differences by sex.

$\mathrm{t}$ is well known that males are disadvantaged in terms of many health outcomes, particularly cardiovascular and respiratory outcomes. Overall life expectancy in the UK is consistently lower for men than women (1). The current live birth ratio in England and Wales is 1.05 for boys:girls, and yet the stillbirth rate is approximately $2 \%$ higher in boys than girls (2). The mean birth weight is higher in boys than girls (3), but boys are more likely to be born prematurely (4).

Male vulnerability has been previously demonstrated in infants born with very low birth weight or born very prematurely. As compared with females, males had more postnatal complications, including lower Apgar scores, a greater need for supplemental oxygen, higher rates of respiratory distress syndrome, more pulmonary interstitial emphysema, and higher overall perinatal mortality rates $(5-8)$. There are some clues as to why preterm boys fare worse than preterm girls as advanced lung maturity by approximately $1 \mathrm{wk}$ is reported in girls during the last 2 mo of pregnancy (9). Furthermore, male fetuses are exposed to higher levels of androgen and Mullerian inhibiting substance, which adversely affect surfactant production $(10,11)$. Thus, it is reasonable to conclude that lung immaturity in premature boys contributes to their poorer outcomes.

The now routine use of antenatal corticosteroids and postnatal surfactant have been associated with a substantial improvement in survival $(8,12,13)$. It may be therefore that there are less marked differences in outcome for boys and girls following the introduction of these treatments. When, however, we followed up a group of very prematurely born infants in whom corticosteroids and surfactant were almost universally used, we found that male sex was a risk factor for poorer lung function (14), increased respiratory morbidity (15), and poorer neurological function overall (16). In this article, we build on our previous analyses to try to understand causal pathways by first exploring sex differences in a range of neonatal outcomes and then using statistical models to sequentially adjust later outcomes for neonatal differences. In this way, we sought to determine if the observed later effects of male sex could be attributed to the poorer neonatal profile or whether there is evidence for an intrinsic male effect.

This study aimed to answer the following questions: (i) Do boys have worse neonatal outcome than girls? (ii) Do boys have worse longer term respiratory and neurological outcomes than girls? (iii) Given the findings for neonatal outcomes, does adjustment for identified risk factors attenuate the effects observed at follow-up?

\section{RESULTS}

The data from 797 infants were included in this analysis, 428 boys and 369 girls; 205 (26\%) died before discharge. There were no significant differences by sex for any maternal characteristic (Table 1).

\footnotetext{
'Division of Health and Social Care Research, King's College London, London, UK; ${ }^{2}$ Department of Primary Care and Population Health, University College London, London, UK; ${ }^{3}$ Institute for Women's Health, University College London, London, UK; ${ }^{4}$ Department of Child Health, St George's University of London, London, UK; ${ }^{5}$ Division of Asthma, Allergy and Lung Biology, King's College London, London, UK. Correspondence: Janet L. Peacock (janet.peacock@kcl.ac.uk)
} 
Table 1. Maternal factors by sex: means or percentages and $P$ values for difference

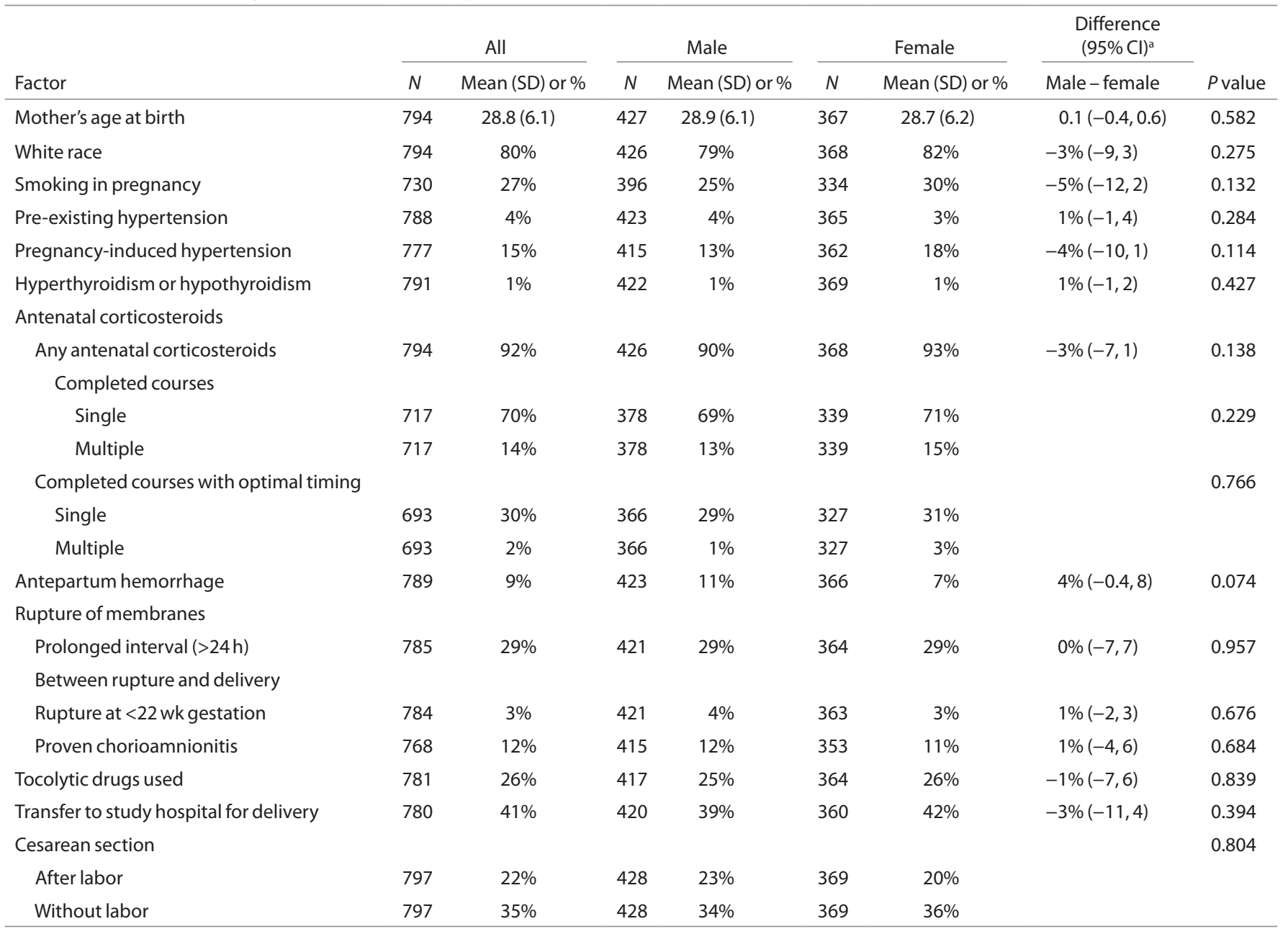

Maximum sample $=797$ (428 males, 369 females).

aDifferences as proportions have been presented where calculable. All analyses adjusted for clustering in multiple births (19).

Table 2. Infant factors by sex: means or percentages and $P$ values for difference

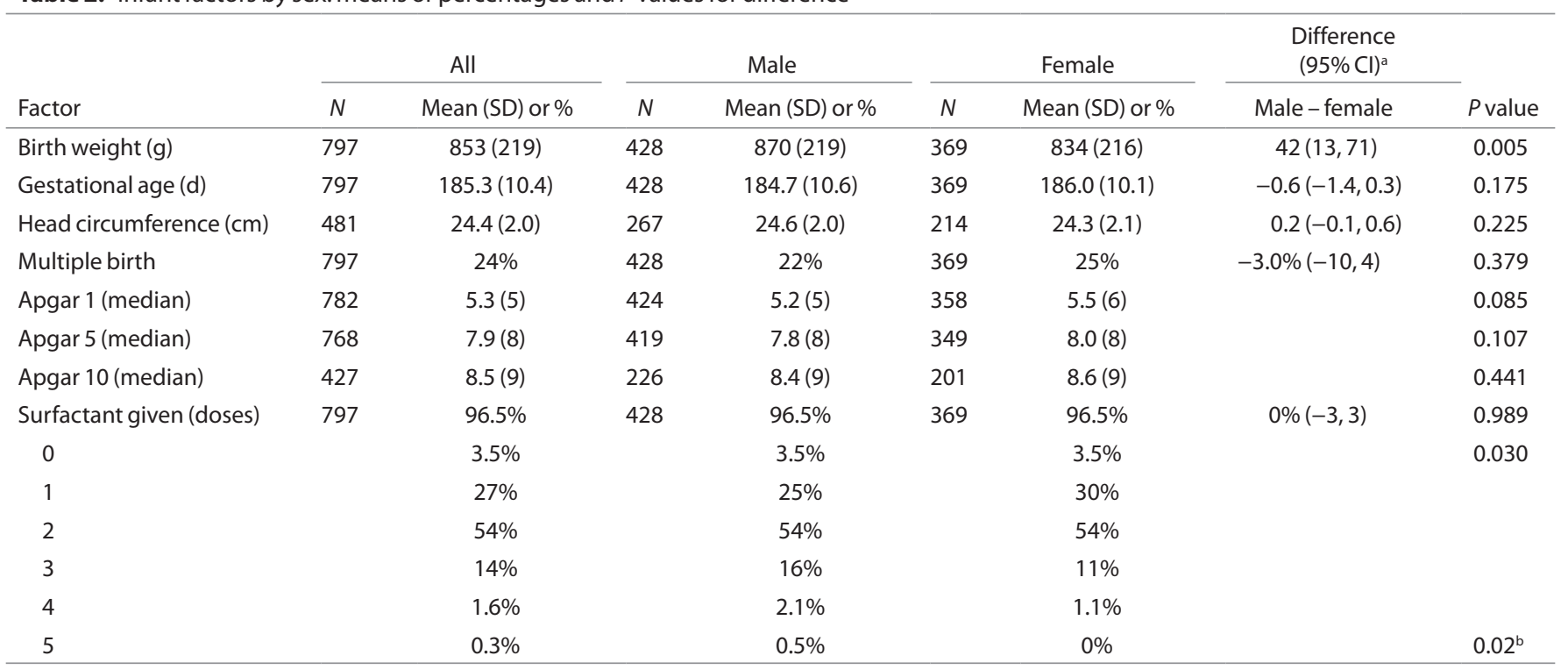

Maximum sample $=797$ (428 males, 369 females).

aifferences as proportions have been presented where calculable. All analyses adjusted for clustering in multiple births (19). ${ }^{\mathrm{b}}$ Test for trend. 
Two of the eight infant factors differed significantly by sex (Table 2): Mean birth weight was $42 \mathrm{~g}$ higher in boys than girls, and although similar proportions of boys as girls received any surfactant, boys received significantly more doses.

The primary outcome of the trial, death or oxygen dependency, varied significantly by sex $(72 \%$ in boys vs. $61 \%$ in girls; Table 3). In addition, the component parts of the composite outcome, death and oxygen dependency, each showed higher rates in boys: $28 \%$ versus $23 \%$ for death, and $44 \%$ versus $38 \%$ for oxygen dependency. After adjustment for birth weight and gestation, the overall difference between the primary outcome rate in boys and girls increased further from 11 to 15 percentage points. Among infants who died, boys lived significantly longer than girls. This difference was even more marked after adjustment for birth weight and gestation, showing a 50\% longer survival among boys who ultimately died as compared with girls who died. Hospital stay was longer on average in boys than girls, and particularly so after adjustment, showing that boys' length of stay was approximately $13 \%$ longer than that of girls. Pulmonary hemorrhage was significantly more common in boys, with a 5 percentage point difference, equivalent to a $50 \%$ increase in rate among boys as compared with girls. The use of postnatal steroids was significantly greater in boys than girls with a difference of 17 percentage points. Nearly twice as many boys as girls had a major cranial abnormality, and, although this difference was less after adjustment, it remained significant and substantial at 6 percentage points.

Table 4 shows that disability (either severe or otherwise and based on parental assessment (ref. 16)) was present at 24-mo follow-up in 53\% of boys as compared with $39 \%$ of girls. This difference remained significant after adjustment for birth weight, gestational age, and oxygen dependence at $36 \mathrm{wk}$

Table 3. Neonatal outcomes by sex: unadjusted and adjusted for birth weight and gestational age

\begin{tabular}{|c|c|c|c|c|}
\hline \multirow[b]{2}{*}{ Outcome } & \multirow[b]{2}{*}{ Male } & \multirow[b]{2}{*}{ Female } & \multirow{2}{*}{$\begin{array}{c}\text { Difference }(95 \% \mathrm{Cl})^{\mathrm{a}} \\
\text { (Male - female })^{2}\end{array}$} & \multirow{2}{*}{$\frac{\text { Adjusted difference }(95 \% \mathrm{Cl})^{\mathrm{b}}}{(\text { Male }- \text { female })}$} \\
\hline & & & & \\
\hline Died/oxygen dependent at 36 wk & $72 \%(308 / 428)$ & $61 \%(225 / 369)$ & $11 \%(4,18)$ & $15 \%(7,22)$ \\
\hline Oxygen dependent at $36 \mathrm{wk}$ & $44 \%(189 / 428)$ & $38 \%(139 / 369)$ & & \\
\hline Died & $28 \%(119 / 428)$ & $23 \%(86 / 369)$ & & \\
\hline Hospital stay $(d)^{c}$ & $97(N=308)$ & $86(N=281)$ & $1.12(1.06,1.18)$ & $1.13(1.08,1.18)$ \\
\hline Air leak & $19 \%(81 / 428)$ & $15 \%(55 / 369)$ & $4 \%(-2,9)$ & $3 \%(-2,8)$ \\
\hline Pulmonary hemorrhage & $15 \%(63 / 422)$ & $10 \%(36 / 363)$ & $6 \%(1,10)$ & $5 \%(1,9)$ \\
\hline Postnatal steroids & $37 \%(134 / 364)$ & $21 \%(65 / 316)$ & $16 \%(10,23)$ & $17 \%(10,25)$ \\
\hline PDA & $33 \%(140 / 426)$ & $34 \%(126 / 367)$ & $-1 \%(-8,5)$ & $-3 \%(-10,3)$ \\
\hline NEC & $11 \%(47 / 423)$ & $9 \%(33 / 364)$ & $2 \%(-2,6)$ & $2 \%(-2,7)$ \\
\hline
\end{tabular}

Maximum sample $=797$ (428 males, 369 females).

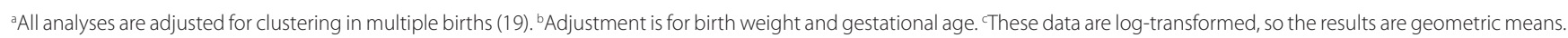
The differences are expressed as ratios of geometric means. Results in bold indicate $P<0.05$.

$\mathrm{Cl}$, confidence interval; NEC, necrotizing enterocolitis; PDA, patent ductus arteriosus; ROP, retinopathy of prematurity.

Table 4. 24-Month follow-up outcomes $(N=366)$

\begin{tabular}{|c|c|c|c|c|c|c|}
\hline Outcome & Male & Female & Difference & Adjusted difference ${ }^{a}$ & Adjusted difference ${ }^{b}$ & Adjusted difference ${ }^{c}$ \\
\hline Any disability ${ }^{d}(n=363)$ & $53 \%$ & $39 \%$ & $14 \%(3,24)$ & $15 \%(4,25)$ & $15 \%(3,27)$ & $12 \%(-1,25)$ \\
\hline Cognitive delaye $(n=288)$ & $35 \%$ & $19 \%$ & $15 \%(4,26)$ & $17 \%(6,28)$ & $14 \%(2,26)$ & $8 \%(-4,22)$ \\
\hline Wheeze $(n=354)$ & $41 \%$ & $33 \%$ & $8 \%(-2,19)$ & $9 \%(-2,19)$ & $9 \%(-2,19)$ & $9 \%(-2,20)$ \\
\hline Any chest medicines $(n=363)$ & $62 \%$ & $52 \%$ & $10 \%(-1,20)$ & $11 \%(-0,21)$ & $10 \%(-1,21)$ & $12 \%(0,23)$ \\
\hline
\end{tabular}

Maximum number of children with follow-up data $=70 \%$ of UK surviving children (366/521).

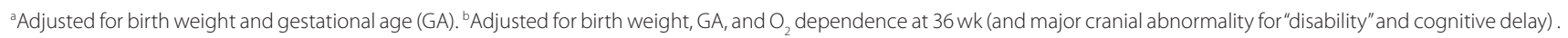
"Neurological outcomes are adjusted for birth weight, GA, O dependence at 36 wk, major cranial abnormality, length of hospital stay after birth, age at 24-mo assessment, and weight standard deviation score at 24 mo; respiratory outcomes are adjusted for birth weight, $\mathrm{GA}_{2} \mathrm{O}_{2}$ dependence at 36 wk, multiple birth, and older sibling aged $<5$. All analyses adjusted for clustering in multiple births (19). ${ }^{\mathrm{d} A n y}$ disability is derived from parent questionnaire as detailed in Marlow (16). ${ }^{e}$ Cognitive delay was from the Parent Report of Children's Abilities (PARCA) score $<49$, which corresponds to Mental Development Index $\leq 70$ (18). Results in bold indicate $P<0.05$. 


\section{Articles | Peacocket al.}

postmenstrual age, but the difference was smaller after further adjustment for length of hospital stay, age at assessment, and weight standard deviation score, and became nonsignificant. There were similar findings for cognitive delay, which was based on parental report (35\% vs. 19\%; Table 4). Cough (a positive parental response to "does your child suffer from coughing") and wheeze (a positive response to the question "does your child suffer from wheezing") were more common in boys than girls at 24-mo corrected age but not significantly so (52\% vs. $47 \%$ and $41 \%$ vs. $33 \%$, respectively). More boys than girls had used bronchodilators or inhaled steroids ( $48 \%$ vs. $31 \%$ ) and differences remained significant after adjustment (Table 4). The full table of results for 24-mo follow-up outcomes is given in Supplementary Table S1 online.

\section{DISCUSSION}

Consistent with the findings of others, we have shown that very prematurely born boys had markedly higher rates of adverse neonatal outcomes. The combined outcome, death or oxygen dependency, showed an 11 percentage point difference between boys and girls. The excess in males was present for the two constituent parts of the composite outcome-death and oxygen dependency. Boys died later, with median age of death of $7 \mathrm{~d}$ (range $0-320$ ), as compared with girls, with a median of $4 \mathrm{~d}$ (range $0-185$ ). However, the interquartile ranges were very similar for boys and girls (2-19, 1-20), suggesting that there were more outlier "late" deaths among the boys. Boys had substantially higher rates of pulmonary hemorrhage, stayed in hospital longer, and were given more postnatal steroids, reflecting their increased respiratory morbidity as compared with girls. These findings were hardly changed by adjustment for birth weight and gestational age. Brothwood (1986) reported a similar difference in survival of 14 percentage points among 271 very-low-birth-weight infants born during 1980-82 in London (5). Stevenson's multicenter study of 6,738 verylow-birth-weight infants in 1991-1993 in the United States reported mortality rates of $22 \%$ for boys and $15 \%$ for girls (6). The EPICure study reported on 811 extremely preterm infants (23-25 wk gestational age) admitted to the UK and Republic of Ireland neonatal units in 1995 and found an odds ratio for death of 1.31 for male sex (7). Survival among very prematurely born infants has increased steadily in the past 20 years (8), and so the interpretation of absolute mortality rates in boys and girls over time is problematic. The sex differences, however, in mortality observed in this study of births during 1998-2001 are similar in relative size to those of previous studies, and so it appears that the use of antenatal steroids and postnatal surfactant, although improving survival overall, has not narrowed the gap in mortality rates between boys and girls.

Although pulmonary outcomes have been most widely reported as showing differences between boys and girls, neurological outcome was also substantially worse in boys than girls in our study, in keeping with some previous reports $(5,6)$ but not others (7). The observed sex differences in neurological outcome were not explained by major cranial abnormality. Cerebral ultrasound (CUS) is a good way of detecting major cranial hemorrhagic or extensive ischemic lesions, but it is not good at detecting the more subtle aspects of brain injury that are apparent in more recent MRI studies. Boys may have a higher risk of brain injury through these major routes as in EPICure, which showed that severe disability and cerebral palsy were more common in boys (21). However, when these issues are taken away, boys still have lower developmental scores than girls. Thus, CUS only helps in the causal pathway for those with major brain injury but does not detract from sex differences found widely in very preterm children.

The maternal data show no evidence of differences between the sex groups, and so it seems very unlikely that maternal factors are responsible for the sex differences observed. The multifactorial analyses included birth weight and gestation as confounders, and those analyses either slightly increased the sex difference or slightly reduced it but did not remove it.

Our study is limited in that it was a secondary analysis of trial data and the follow-up rate was low at $70 \%$. However, although the children who were lost to follow-up were less likely to have had chronic lung disease, this was not statistically significant and rates of major cranial abnormality were similar in those followed-up and those lost. Our outcomes for disability and cognitive function were based on parental questionnaires, which is a disadvantage, but the parental cognitive function questionnaire (PARCA) has been validated against the Bayley Scales of Infant Development-II (18) and comprises statements of development taken from the Bayley and the MacArthur language test. It has been widely used as a surrogate for doing a formal Bayley assessment in large studies including one to be published in the NEJM in the near future. Although we were able to adjust for a range of neonatal factors, our study was limited by the availability of data already collected and it is possible that further adjustment would affect the differences reported.

Male/female differences in lung function have been demonstrated in term (22) and preterm (23) infants with males tending to fare worse than females. A study of lung profiles showed sex differences in normal pregnancies (9). Four indices (lecithin/sphingomyelin ratios, percentage disaturated lecithin, phosphatidylglycerol, and phosphatidylinositol) were all obtained from amniotic fluid in 164 pregnancies of $30 \mathrm{wk}$ or more to quantify sex differences in surfactant components. All four indices of lung profile showed that the male fetuses had less mature lungs than the females by approximately $1 \mathrm{wk}$ (9). In a more recent and large cohort of births below $31 \mathrm{wk}$ in Canada, Jones et al. showed the familiar male/female differences in acute outcomes but interestingly showed that differences in survival were only apparent for infants born at 24-wk gestation (13). This is consistent with the thesis that the relative male immaturity determines the excess mortality observed in very preterm infants.

Alongside the findings for neonatal endpoints, we observed a higher prevalence of poor neurological and respiratory outcomes at 24 mo for boys compared with girls. Overall these effects did not reduce when adjustment was made for birthweight and gestation, and neither were they reduced when 
additional adjustment was made for oxygen dependency at 36-wk postmenstrual age, mitigating against the hypothesis that for boys their poorer outcome at follow-up was explained by bronchopulmonary dysplasia. Further adjustment for potential confounding factors sometimes reduced effect sizes but not always. Specifically, disability, cognitive delay, and use of respiratory medications remained significantly more common in boys. These findings concur with those of a US NICHD Neonatal Research Network study that also suggested that there is a constitutional difference between the sexes that is not explained by perinatal, neonatal, or postnatal factors (24).

In conclusion, understanding the causal pathway for differences in outcome between boys and girls born very preterm can guide future research as well as indicating when and where to intervene. If the pathway is simply mediated through the neonatal course as we hypothesized, then it is reasonable to focus attention on neonatal care and assess the need for intervention on the basis of neonatal outcome regardless of sex. If, however, as these results suggest, there is separate male vulnerability, then alternative causal pathways need to be assessed. Further research is needed to fully understand this important issue.

\section{METHODS}

This study hypothesized that the effects of male sex on later outcomes could be attributed to the poorer neonatal profile and thus sought to find evidence in favor or against this assertion.

\section{Source of Data}

The United Kingdom Oscillation Study (UKOS) was a multicenter randomized controlled trial in infants born at 23-28-wk gestational age requiring endotracheal intubation from birth and ongoing intensive care. The recruited infants were randomized to either high-frequency oscillation or conventional mechanical ventilation within $1 \mathrm{hr}$ of birth. Seven hundred ninety-seven infants were recruited into the trial between August 1998 and January 2001 (17).

\section{Variables Considered}

Maternal factors and birth factors were analyzed as possible prognostic factors for neonatal outcome. All factors were initially compared in boys and girls.

Maternal factors included age, white/nonwhite ethnicity, smoking in pregnancy, pre-existing or pregnancy-induced hypertension requiring treatment, thyroid disease, antenatal corticosteroids, antepartum hemorrhage, rupture of membranes, use of tocolytic drugs, predelivery transfer-in, and cesarean section. Birth factors included birthweight, gestational age, head circumference, singleton/multiple birth, and Apgar score at 1,5 , and $10 \mathrm{~min}$.

Neonatal outcomes included death or oxygen dependency at 36-wk postmenstrual age, age at death, length of hospital stay, air leak, pulmonary hemorrhage, patent ductus arteriosus (PDA) requiring treatment, major cranial ultrasound abnormality (including intraventricular hemorrhage with ventricular dilation or any parenchymal hemorrhage), retinopathy of prematurity (stage $2+$ ), hearing test outcome from a national screening test which comprises ABR and evoked acoustic emissions, and necrotizing enterocolitis (NEC; systemic illness with evidence of intramural gas on AXR and/or histological evidence at laparotomy) with surfactant use and postnatal steroids treated as possible prognostic factors.

Follow-up outcome data were obtained at 24-mo corrected age. Some follow-up data have already been reported but sex differences were not the main focus of those analyses (16). In addition to exploring neonatal factors, we now present a sequential analysis to show how sex differences in infant outcomes change (or not) when adjustment is made for important neonatal factors.

Follow-up data analyzed included outcomes obtained at $24 \mathrm{mo}$ using a parent-completed neurodevelopmental questionnaire which recorded information on health status and anthropometry (16). From these questions, the outcomes "severe disability", "other disability," or "no disability" were derived. "Severe disability" is at least one extreme response in one of the following clinical domains: neuromotor, vision, hearing, communication, or other physical disabilities; "no disability" is a normal or missing response to all clinical domains, as described in Marlow (2006) (16). A parental assessment of cognitive development (PARCA) was used that has been validated against Bayley Scales of Infant Development-II and comprises statements of development taken from the Bayley and the MacArthur language tests (18). For PARCA, a score of $<49$ is equivalent to a Bayley Mental Development Index $\leq 70$ (18) and is therefore used as an indicator of cognitive delay. In addition, respiratory symptoms and medication use outcomes at 24 mo, which had previously been found to exhibit sex differences $(15,16)$, were reanalyzed to adjust sequentially for neonatal factors.

\section{Statistical Analyses}

Random-effects (mixed) models were used throughout to allow for the clustering among multiple births (19) with normal or binomial errors for continuous or binary data, respectively. Continuous data were transformed where necessary. This modeling approach allowed estimation of the adjusted differences between boys and girls for maternal and infant factors with 95\% confidence intervals and $P$ values. Adjusted absolute differences are reported here rather than relative differences such as odds ratios, as they are more informative in quantifying the extent of differences between boys and girls.

To tease out the effect of sex per se, the models for neonatal outcomes were adjusted for birth weight and gestational age. Trial treatment group was not included in modeling because this factor had previously been shown to be nonsignificant with effect estimates close to null (17).

Further adjustment was conducted in three stages to show how differences observed at follow-up might be explained by (i) birth factors (birth weight and gestational age), (ii) birth and neonatal outcomes data (birth weight and gestational age and oxygen dependency at 36-wk postmenstrual age, and major cranial abnormality for disability and cognitive delay), and (iii) all of these variables plus others that had previously been found to be important predictors of the followup outcomes (as stated in the footnotes of each table).

All analyses were conducted using Stata (20).

The UKOS trial was approved by the London South Thames Multicentre Research Ethics Committee. Parents gave informed written consent for their infants to take part.

\section{SUPPLEMENTARY MATERIAL}

Supplementary material is linked to the online version of the paper at http://www.nature.com/pr

\section{STATEMENT OF FINANCIAL SUPPORT}

The United Kingdom Oscillation Study was funded by the UK Medical Research Council.

\section{REFERENCES}

1. Office for National Statistics 2006 Focus on Gender. Available at: http:// www.ons.gov.uk/ons/publications/re-reference-tables.html? edition= tcm\%3A77-51142 (accessed September 25, 2011).

2. Office for National Statistics 2007 Birth statistics 2006 series FM1 No 35. Available at: http://www.ons.gov.uk/ons/rel/vsob1/birth-statistics-england-and-wales--series-fm1-/no--35--2006/index.html (accessed September 25, 2011).

3. Centre for Longitudinal Studies 2006 The Millenium Studies. Available at: http://www.cls.ioe.ac.uk/studies.asp?section=000100020001 (accessed September 25, 2011). 


\section{Articles Peacocket al.}

4. Zeitlin J, Saurel-Cubizolles MJ, De Mouzon J, et al. Fetal sex and preterm birth: are males at greater risk? Hum Reprod 2002;17:2762-8.

5. Brothwood M, Wolke D, Gamsu H, Benson J, Cooper D. Prognosis of the very low birthweight baby in relation to gender. Arch Dis Child 1986;61:559-64.

6. Stevenson DK, Verter J, Fanaroff AA, et al. Sex differences in outcomes of very low birthweight infants: the newborn male disadvantage. Arch Dis Child Fetal Neonatal Ed 2000;83:F182-5.

7. Costeloe K, Hennessy E, Gibson AT, Marlow N, Wilkinson AR. The EPICure study: outcomes to discharge from hospital for infants born at the threshold of viability. Pediatrics 2000;106:659-71.

8. Hack M, Fanaroff AA. Outcomes of children of extremely low birthweight and gestational age in the 1990's. Early Hum Dev 1999;53: 193-218.

9. Fleisher B, Kulovich MV, Hallman M, Gluck L. Lung profile: sex differences in normal pregnancy. Obstet Gynecol 1985;66:327-30.

10. Catlin EA, Powell SM, Manganaro TF, et al. Sex-specific fetal lung development and müllerian inhibiting substance. Am Rev Respir Dis 1990;141:466-70.

11. McMillan EM, King GM, Adamson IY. Sex hormones influence growth and surfactant production in fetal lung explants. Exp Lung Res 1989;15:167-79.

12. Jobe AH, Mitchell BR, Gunkel JH. Beneficial effects of the combined use of prenatal corticosteroids and postnatal surfactant on preterm infants. Am J Obstet Gynecol 1993;168:508-13.

13. Jones HP, Karuri S, Cronin CM, et al.; Canadian Neonatal Network. Actuarial survival of a large Canadian cohort of preterm infants. BMC Pediatr 2005;5:40.

14. Thomas MR, Marston L, Rafferty GF, et al. Respiratory function of very prematurely born infants at follow up: influence of sex. Arch Dis Child Fetal Neonatal Ed 2006;91:F197-201.
15. Greenough A, Limb E, Marston L, et al. Risk factors for respiratory morbidity in infancy after very premature birth. Arch Dis Child Fetal Neonatal Ed 2005;90:F320-3.

16. Marlow N, Greenough A, Peacock JL, et al. Randomised trial of high frequency oscillatory ventilation or conventional ventilation in babies of gestational age 28 weeks or less: respiratory and neurological outcomes at 2 years. Arch Dis Child Fetal Neonatal Ed 2006;91:F320-6.

17. Johnson AH, Peacock JL, Greenough A, et al.; United Kingdom Oscillation Study Group. High-frequency oscillatory ventilation for the prevention of chronic lung disease of prematurity. N Engl J Med 2002;347:633-42.

18. Johnson S, Marlow N, Wolke D, et al. Validation of a parent report measure of cognitive development in very preterm infants. Dev Med Child Neurol 2004;46:389-97.

19. Marston L, Peacock JL, Yu K, et al. Comparing methods of analysing datasets with small clusters: case studies using four paediatric datasets. Paediatr Perinat Epidemiol 2009;23:380-92.

20 Stata version 10.02008 Statacorp Texas USA.

21. Wood NS, Costeloe K, Gibson AT, et al.; EPICure Study Group. The EPICure study: associations and antecedents of neurological and developmental disability at 30 months of age following extremely preterm birth. Arch Dis Child Fetal Neonatal Ed 2005;90:F134-40.

22. Jones M, Castile R, Davis S, et al. Forced expiratory flows and volumes in infants. Normative data and lung growth. Am J Respir Crit Care Med 2000;161:353-9.

23. Hoo AF, Henschen M, Dezateux C, Costeloe K, Stocks J. Respiratory function among preterm infants whose mothers smoked during pregnancy. Am J Respir Crit Care Med 1998;158:700-5.

24. Hintz SR, Kendrick DE, Vohr BR, Kenneth Poole W, Higgins RD; Nichd Neonatal Research Network. Gender differences in neurodevelopmental outcomes among extremely preterm, extremely-low-birthweight infants. Acta Paediatr 2006;95:1239-48. 